\title{
Understanding the Use of Temporal Expressions on Persian Web Search
}

\author{
Behrooz Mansouri \\ Telecommunication Research Center \\ Tehran, Iran \\ b.mansouri@itrc.ir \\ Ricardo Campos \\ Polytechnic Institute of Tomar Smart Cities \\ Research Center \\ LIAAD - INESC TEC \\ Portugal \\ ricardo.campos@ipt.pt
}

Web Azma Lab, Information Technology Faculty, Iran

\author{
Mojgan Farhoodi \\ Web Azma Lab, Information \\ Technology Faculty, Iran \\ Telecommunication Research Center \\ Tehran, Iran \\ farhoodi@itrc.ir
}

\author{
Mohammad Sadegh Zahedi \\ Web Azma Lab, Information Technology Faculty, Iran \\ Telecommunication Research Center \\ Tehran, Iran \\ s.zahedi@itrc.ir
}

\begin{abstract}
The development of information retrieval algorithms and temporal information retrieval ones has been extensively carried out over the last few years. While several studies have been conducted, most of these researches relate to English, leading to a lack of knowledge in several other important languages. This includes the Persian one. In this work, we aim to shorten this gap by contributing, disseminating and enlarging the knowledge we have on temporal information retrieval aspects in Persian, which is one of the dominant languages in the Middle East, widely spoken in several countries. To achieve this objective, we propose to understand the use of temporal expressions on a large-scale Persian search engine query log consisting of $27 \mathrm{M}$ queries. In particular, we focus on explicit (e.g., June 2017) and relative temporal expressions (e.g., tomorrow) and try to understand (1) how often temporal expressions are used in web queries; (2) which type of temporal expressions (Date, Time, Duration and Set) are commonly used; (3) to which time (past, current or future) do temporal expressions mostly refer to; (4) to which category they often belong; (5) how often do user's reformulate their queries by adding temporal expressions; and (6) how using temporal expressions affects user's satisfaction. We believe that answering these questions may be beneficial for a large number of tasks including, user's behavior understanding and search engines' improvement effectiveness.
\end{abstract}

\section{ACM Reference Format}

Behrooz Mansouri, Mohammad Sadegh Zahedi, Ricardo Campos, Mojgan Farhoodi, Alireza Yari. 2018. Understanding the Use of Temporal Expressions on Persian Web Search. In WWW'18 Companion: The 2018 Web Conference, April 23-27, 2018, Lyon, France. ACM, NY, NY, USA, 6 pages. https://doi.org/10.1145/3184558.3191635

This paper is published under the Creative Commons Attribution-NonCommercialNoDerivs 4.0 International (CC BY 4.0) license. Authors reserve their rights to disseminate the work on their personal and corporate Web sites with the appropriate attribution. WWW'18 Companion April 23-27, 2018, Lyon, France.

(c) 2018 IW3C2 (International World Wide Web Conference Committee), published under Creative Commons CC BY 4.0 License.

ACM ISBN 978-1-4503-5640-4/18/04.

DOI: https://doi.org/10.1145/3184558.3191635

\section{KEYWORDS}

Temporal Queries, Query Log Analysis, Web Search

\section{INTRODUCTION}

Over the last few years, temporal queries have been gaining an increasing importance when it comes to web search, leading search engines to improve their search retrieval effectiveness. This can be done, either by giving higher ranks to the documents related to the mentioned time in the query (be it explicit or implicit) or by suggesting related or similar temporal ones. For example, a user who issues the query "London Olympics", which was held in the year 2012, should be given more documents related to this time-period. If the user is not satisfied with the search results, he or she may add the additional temporal expression " 2012 " to the initial query, provided it knows the year in which London Olympics took place. Otherwise, one may expect the search engine to suggest the query "London Olympics 2012 ". However, this can also only be done, if the search engine has the ability to detect the implicit temporal intent behind a query, which to worsen may refer to more than one single time point. This problematic, has been extensively tackled over the last few years with substantial work involving the use of implicit temporal queries and their related ambiguity. In particular, Metzler et al. [19], used query logs to investigate implicitly year qualified queries, by investigating how strongly these queries are associated with several different years. The work by Campos et al. [9] used the temporal information within the k-top retrieved web snippets for a query to identify its most relevant time periods. The same authors [7] proposed to classify implicit temporal queries according to their temporal ambiguity, using information extracted from web snippets. More recently, Mansouri et al.[18] extended these categories by proposing a new taxonomy where queries are classified into one of four classes: temporal unambiguous, unpredictable temporal ambiguous queries, predictable event-related temporal ambiguous queries and predictable commemorative temporal ambiguous ones. Another type of temporal queries, studied in $[10,17]$, are related to seasonal events, and are characterized by the occurrence of seasonal spikes in search logs. One such example is the query "Halloween", which tends to occur more 
often when the 31th of October approaches. Despite a noticeable number of studies on implicit temporal queries approaches, only a few works have studied the explicit temporal ones. These, usually rely on query log analysis, a well-known research topic in information retrieval and data mining, which studies how query logs may be used to understand user's behavior and improve search engines' effectiveness. Eickhoff et al. [11], for instance, studied query logs to understand how the language and the search behavior of a user on a topic changes over time by considering developments of expertise at session level. Bendersky et al. [4] in turn, brought attention to long queries and studied information needs behind them. They showed that user click behavior is correlated with query length. Another work is that of Rieh [21] who investigated the patterns of multiple web query reformulations. Although several works have considered the importance of temporal expressions in web queries, few works have considered to study their importance within explicit temporal queries, with Nunes et al. [20] being the most noticeable one. In their work the authors studied the use of temporal expressions in web search based on two AOL query log datasets, and came to the conclusion that only $1.5 \%$ of the queries had temporal expressions. As a further outcome they also report that most of the queries are related to the categories of "Auto", "Sports" and "News \& Society", with people mostly using temporal expressions of the type "year" to refer to current and past events. In this work, we will provide a similar study. However, unlike Nunes et al. [20], we will study how both explicit and relative temporal expressions are used in Persian web searches. To do this, we rely on two-year query log records of the Persian search engine, Parsijoo ${ }^{1}$. In addition to the analysis of Nunes et al. [20], we investigate how temporal expressions are used for query reformulation with a more elaborated notion of user search session, and show how they affect user's search satisfaction. Furthermore, we study, the use of temporal expressions according to the TIMEX3 annotation [23]. To this regard, we consider four types of temporal expression: Date (e.g., June 2017; tomorrow), Time (e.g., 8 a.m. Friday), Duration (e.g., 3 hours) and Set (e.g., once a week). Another contribution of our study is that we explore how temporal expressions are used within each query category. That is, we investigate whether queries of a particular category (say "Business and Finance") are mostly focused on the past, present or future time. By knowing this, search engines may, for example, give higher emphasis to documents referring to a particular time-period, as opposed to promoting documents with an irrelevant temporal focus or with no temporal nature at all. One such strategy will certainly contribute to improve search results effectiveness. In order to answer to these questions, we aim to understand to which extent and how temporal expressions are used in Persian web search queries. Lay based on this, we formulate the following research questions:

RQ1: How often (explicit or relative) temporal expressions are used in web queries?

\footnotetext{
${ }^{1}$ https://parsijoo.ir
}

RQ2: Which type of temporal expressions (Date, Time, Duration and Set) are commonly used?

RQ3: To which time (past, current or future) do temporal expressions mostly refer to?

RQ4: Which categories are most prevalent in terms of the use of temporal expressions?

RQ5: How often do users reformulate their queries by adding temporal expressions to their initial query?

RQ6: How using temporal expressions affects user's satisfaction?

Answering these questions may be kind of useful for tasks such as query auto completion, query suggestion, document reranking or temporal diversification [2,5,6,10,14,15] A fully detailed description on temporal information retrieval applications can be found in the survey of Campos et al.[8].

The remainder of this paper is organized as follows. Section 2 presents our experimental setting. Section 3 describes the different results of our experiments. Finally, Section 4 provides some conclusions and outlines future work.

\section{EXPERIMENTAL SETUP}

To conduct our analysis, we resort to a Persian search engine query log consisting of $27 \mathrm{M}$ queries and corresponding users' interaction with the search engine. Persian is an Indo-European language, which is one of the dominant languages in the Middle East widely spoken in several countries like Iran, Tajikistan and Afghanistan. Many researches in NLP and information retrieval concern this language $[1,17,25,26]$.

For this study, we considered two-year query log records (ranging from March 2015 to March 2017) and keep, for further analysis, the following information: Query, Query issue time (which will be used to understand whether a query refers to the past, present or future), Session Id (which will be used in the query reformulation process) and the Clicked URL (which will be used to measure the user's satisfaction). We then apply our recently developed Persian time tagger tool, Parstime [16] to identify the explicit and relative temporal signals over each query. The field of temporal taggers is a mature one with several taggers being developed over the last few years. We emphasize the works of Verhagen et al. [24] who, as part of the TARSQI developed GUTime, an English temporal tagger focused on temporal question answering systems, and Strötgen et al. [22] who developed one of the most well-known temporal taggers for English and also Arabic, Italian, Spanish and Vietnamese languages.

\section{EXPERIMENTAL RESULTS}

In this section we will present the results of our analysis on the use of temporal expressions in web searches and their related research questions.

\subsection{Use of temporal expressions}

As the first part of our analysis, we will explore how frequently temporal expressions are used in web searches. Same as Nunes 
et al. [20], our experiment also reveals that queries with temporal expressions (either explicit or relative) are relatively rare and account for only $1.8 \%$ of the total queries. After removing the duplicate queries, temporal expressions still account for only $1.9 \%$ of the web queries, which given the high number of queries studied, still represents $513 \mathrm{k}$ queries. This answer our first research question. A more detailed analysis also allows us to conclude that $49.8 \%$ of the temporal queries are explicitly defined as so by the users. In contrast, $50.2 \%$ of the temporal queries are relative, meaning that a reference time, such as the query issue time, is needed in order to understand to which time they refer to.

\subsection{Types of temporal expressions commonly used}

Next, we analyze which types of temporal expressions were commonly used. We considered the four categories introduced in TIMEX3 [23] annotation: Date (e.g., June 2017, tomorrow), Time (e.g., 8 a.m. Friday), Duration (e.g., 3 hours) and Set (e.g., once a week). The results obtained show that $73.7 \%$ of the temporal queries issued comply with the type Date, $19.1 \%$ with the type Time, $4.4 \%$ of the temporal queries are expressed as Duration and only a marginal number of $2.8 \%$ are expressed as Sets. This reveals a notable tendency of users to make use of dates when expressing their temporal intents, as opposed to other forms of temporality. This answers our research question number 2 .

Going into detail, Table 1 indicates the top-5 most frequent temporal expression patterns which account for $80.6 \%$ of the total queries with temporal expressions. This resembles a Zipf distribution and the behavior of many problems in information retrieval (such as stopwords), where a reduced number of items represents a big part of the overall collection, whereas a huge number accounts for a reduced part. A detailed analysis of the results presented in the table reveals an expected tendency of users to prefer years when expressing their temporal intents, meaning that people often look for events or happenings for which they already have a temporal knowledge. Although in a much smaller scale, relative temporal expressions (for which no year is explicitly defined) are still used to a considerable extent, thus suggesting that people often look for events which have occurred or are expected to occur in a very short time-span (e.g., "weather today", "cinema tomorrow"). These however, demand search engines to look at the query issue time and to the publication date of a document in order to understand to which time the relative temporal query refers to.

Table 1. Top-5 most frequent temporal patterns in queries with temporal expressions.

\begin{tabular}{|c|c|c|}
\hline Pattern & Example(s) & Percent \\
\hline Year Expressions & 2017,2016 & $39.8 \%$ \\
\hline Relative Days & Tomorrow, Today & $17.3 \%$ \\
\hline Month + Year & September 2017 & $10.5 \%$ \\
\hline Day + Month & October 21 & $8.3 \%$ \\
\hline Part of Day + Relative Days & Tomorrow Morning & $4.7 \%$ \\
\hline
\end{tabular}

\subsection{Reference time (past, present or future?)}

In this section, we evaluate the reference time to which temporal expressions in queries refer to. This will enable us to answer research question number 3 . To do this, we resort to the query issue time and consider three-time points: past, current and future time. For example, the query "London Olympics 2012", with query issue time on March 2016, refers to the past, "Today's news" refers to the present and "Next week football matches" points to the future. Our analysis indicates that temporal expressions (both explicit and relative) mostly refer to the current time (69.1\% of the temporal expressions). In contrast, past and future related temporal expressions were only used for $16.2 \%$ and $14.7 \%$ respectively.

A more in-depth analysis of the explicit occurrence of years in the query logs is plotted in Figure 1, which spans the period from 1340 to 1400 according to the Jalali calendar (Iran's official calendar). The first ("1340" - 1961 in the Gregorian calendar) refers to death of the famous Iranian clergy "Boroujerdi". The latter (" 1400 ") points to a future date as the time of this writing (2021 in the Gregorian calendar). 6\% of the years were ignored as they referred to years out of the range 1340 to 1400 (with just a few expressions in terms of occurrence).

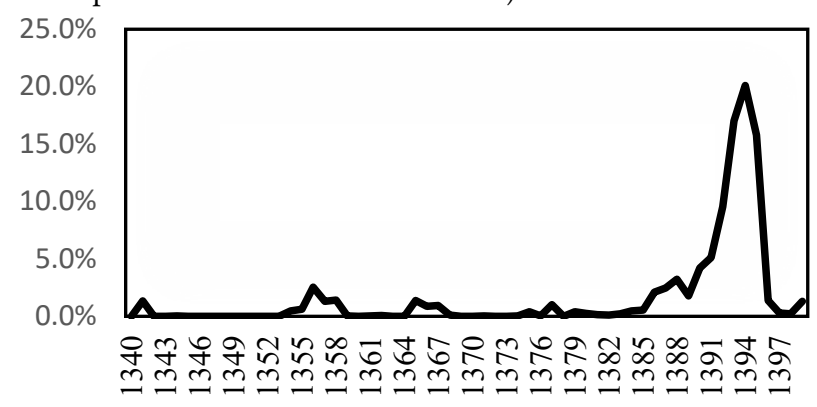

Figure 1. Distribution of years in users' queries.

By looking at the figure, one can conclude that the frequency distribution is mostly skewed to the right with a predominant long tail toward past years, and only a few references to the future. The most frequent years registered in the logs were "1394" and "1395" which refer to the first two years for which the query logs were available (2015 - 2016). These account for almost one third of the total years used in the queries, showing a tendency of users to look for events within a short period of time. When looking at the past, one can also note a slight peak on "1357" (1979 in the Gregorian calendar) and on "1366" (1987 in the Gregorian calendar). Both are used to refer to historical events. The former is due to the Iranian Islamic Revolution, an important historical event in Iran which is officially celebrated every year, thus leading to noticeable amount of temporal expressions. The latter instead, is related to the end of Iran and Iraq war. In contrast, only a few dates refer to the future. These were likely used to search for important events such as the presidential election in both Iran and USA. Future information retrieval is an important and interesting topic in the Information Retrieval area, which was first introduced by Baeza-Yates [3]. 


\subsection{Topic categories}

In this section, we study the distribution of queries with temporal expressions by their topics, in order to answer research question number 4. To do so, we randomly selected a representative sample of 10,000 unique queries with temporal expressions which corresponds to an estimate error smaller than $1 \%$. We then asked three editors to select the best query category for each query. To do this, we considered the categories defined on Yahoo Answers ${ }^{2}$, which covers a variety of different categories, including health, sports, travel and news. An interrater reliability analysis using the Fleiss Kappa statistics [13] was performed to determine consistency among the editors. Overall, the annotators obtained about 0.72 of agreement level, which represents a high agreement between editors. Figure 2 indicates the top 10 categories where temporal expressions were mostly used.

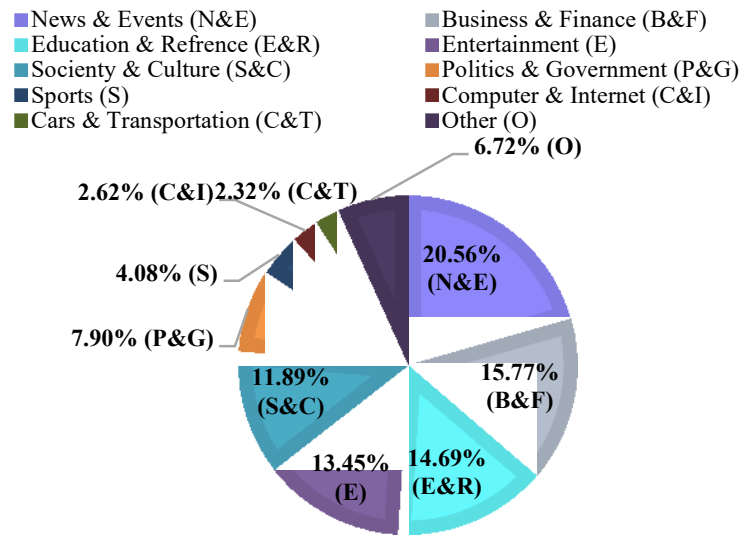

Figure 2. Distribution of temporal queries by their topic category.

As this figure indicates, temporal expressions were mostly used for certain categories, namely "News \& Events (N\&E)", "Business \& Finance (B\&F)" and "Education \& Reference (E\&R)" which account for more than half of all the queries with temporal expressions. This answers research question number 4 . A detailed analysis on each of the three individual categories, reveals that temporal expressions are differently used for each category. For instance, for the "News \& Events" category, users used a variety of different temporal expressions to search for events (e.g., "summer 2016", "2017" and "today"). More to the point, our analysis reveals that the frequency on the use of temporal expressions in queries has a direct relation with the impact of the events, that is, the stronger the impact, the higher the frequency of temporal expressions in queries. A clear picture of this is given in Figure 3, which plots the frequency of years (Jalali calendar) used for the "Earthquake" event. As can be seen from this figure, several different temporal expressions were used by users to search for these events, enabling one to plot a timeline and to get a quick glimpse of it. Two temporal

\footnotetext{
${ }^{2}$ https://answers.yahoo.com/
}

expressions however stand-out particularly. Those with the highest impact and number of deaths: "1382" (2003 in the Gregorian calendar, "Bam earthquake") and 1369 ("1990" in the Gregorian calendar, "Rudbar earthquake"). Although anecdotal, this confirms a possible relation of cause-effect, with people often looking more for events that hugely affect their daily life, that is: higher impact - more searches - more use of temporal expressions.

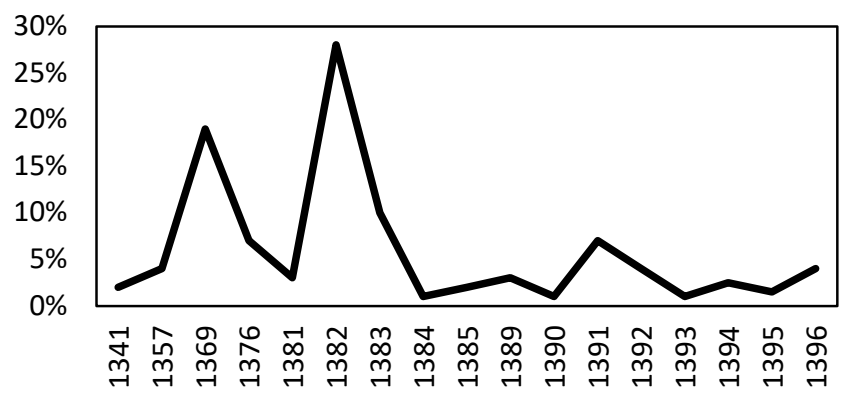

Figure 3. Distribution of years used in the "Earthquake" query.

Another important property of temporal expressions used for "News \& Events" category is that, during the period of the event, users tend to use temporal expressions more related to the present. For instance, during "Rio Olympics", queries such as "today's Olympics results" were dominantly used in searches related to this event, thus suggesting a predominance of users more interested in recent news as opposed to older ones.

In contrast, in the "Business \& Finance" category, $79.4 \%$ of the temporal queries issued make use of relative temporal expressions (e.g., "today" and "tomorrow"), meaning that people are mostly interested in looking for present or near future events. This may be easily explained by the volatility and recency aspects associated to the query category. Finally, for the "Education \& Reference" category, temporal expressions were mostly used to refer to the publication date of books and articles. Therefore, a variety of different temporal expressions, such as "1396", were used in this category.

Based on these results, one can conclude that the temporal expressions used in queries may differ according to the type of category. By knowing this, search engines may eventually decide to promote to the top documents of a specific temporal reference time according to the type and category of the query.

\subsection{Query reformulation with temporal expressions}

In this section, we aim to answer our fifth research question by investigating if temporal expressions were used in the users' initial query or reformulated in subsequent user interactions. To answer this question, we begin by relying on the user's SessionID information, which keeps track of the user's interaction with the search engine, from the moment when the user opens the browser until he/she closes it. Knowing that a user may not close the browser or may issue queries related to different information needs within the same search session, we established a more restricted definition of search session. In this 
definition, a search session is regarded as a sequence of queries issued by the same SessionID within an hour of activity in the search engine. To guarantee that the queries concern the same information need, we require that every two consecutive queries should at least share one common word. Otherwise, we simply consider them as two different search sessions. To study this process, we begin by considering search sessions, where there exists at least one query with temporal expression. We refer to this type of search sessions as temporal search sessions. For example, a search session consisting of the three following queries "Olympics", "Rio Olympics" and "Olympics 2016" is considered a temporal search session since the three queries contains at least one common word ("Olympics") and one temporal expression ("2016"). Based on this definition, we were able to consider a total number of $132.5 \mathrm{k}$ temporal search sessions, (which represents $1.96 \%$ of the total search sessions). The experiments conducted over this data, revealed that in $74.2 \%$ of these search sessions, the initial query already had a temporal expression (e.g., "Olympics 2016"), thus suggesting that a large extension of the users is mostly aware or have been suggested by the search engine a time period related to their information need. A more detailed analysis reveals, however, that this number is boosted by recent dates, as $88.6 \%$ of the initial queries tagged with a date refer to the present, and only a residual number refers to the past $(1 \%)$ or future $(10.4 \%)$. From the side of the users this might be due to the fact that they naturally tend to forget past dates related to historical moments or to ignore future dates related to upcoming events. From the side of the query logs, the fact that only a few users issue queries with past or future dates, impedes search engines to have an enough basis to make user's suggestions. Based on these results one may infer that although a large extent of the initial queries already has a temporal reference, still a huge number of past and future events would benefit from a temporal contextualization. To confirm this assumption, we look at the $25.8 \%$ of the cases, where users temporally reformulate their queries (e.g., Olympics $\rightarrow$ Olympics 2016), to observe their behavior. The results obtained attest to the fact that the vast majority of dates added to the initial query refer to the past $(60 \%)$ or future $(27 \%)$, and that only a residual number refers to the present (13\%). This confirms our assumption and proves the importance of offering a temporal contextualization to the user upon query issue time.

\subsection{The effect of using temporal expressions on users' satisfaction}

Our final analysis was to understand if using the temporal expressions will help users to get better results compared to queries with no temporal expression. To achieve this objective, we measured the user's web search satisfaction. In information retrieval, search satisfaction is defined as the fulfillment of a user's information need [12]. In this work, we define the user's satisfaction measure as a successful search session, where the last interaction between the search engine and the user is a click on an URL. Our assumption is that by ending the search session with a click on an URL the user has found the information he/she was seeking for.
To study this effect, we looked at the $25.8 \%$ of temporal search sessions and attempt to understand whether adding a temporal expression to an initial non-temporal query has led to a final click. The results obtained show that $75.9 \%$ of the search sessions were finished with a click, meaning that, adding a temporal expression to a non-temporal query positively impacts the user's satisfaction. These results were further confirmed by comparing the user's satisfaction in both temporal and nontemporal search sessions (search sessions where queries have no temporal expressions). To conduct this experiment, we randomly selected $132.5 \mathrm{~K}$ non-temporal search sessions, i.e., the same number already considered for the temporal search sessions. This accounts for $4 \%$ of total non-temporal search sessions and corresponds to an estimate error smaller than $1 \%$. The results of our exploration indicate that $78.9 \%$ of the temporal search sessions have satisfied the users, the big majority of which (91\%) ending with a query having a temporal expression. In contrast to this result, only $37.6 \%$ of the web searches were successfully done in non-temporal search sessions. Although a direct causeeffect relation between different queries cannot be proved, we believe this may be due to the existence of temporal expressions in queries. This suggests that adding temporal expression queries, may reduce the query ambiguity and could play an important role to meet users' information needs and search engines to consequently retrieve more relevant documents. This answer our sixth research question and finishes our research work. In the next section, we provide a summary of the results, enlightening and discussing the main points observed.

\subsection{Summary and Discussion of the Results}

In this work we have studied the importance of temporal expressions on Persian web search. The experiments conducted enabled us to conclude that only $1.8 \%$ of the queries contain temporal expressions (of which $50.2 \%$ are relative). Most of these temporal expressions follow the Timex3 date format, of which years and relative expressions are the most common ones. Of particular interest the fact that people are mostly interested in the present, with $69.1 \%$ of the queries with temporal expressions pointing towards the current time. This reflects the fact that both past and future dates are seldom used by users. We speculate that this might be due to the fact that when searching for the past, people might not know the exact date for which to search for. Thus, detecting the temporal nature of a query at first, and suggesting possible related temporal expressions at second, might help user's achieving their seeking objective. In addition, we also found that "News \& Events", "Business \& Finance" and "Education \& Reference" were the dominant categories, when using temporal queries, accounting for more than half of total queries with temporal expressions. We believe that this can be explained by the volatility of the categories, which are extremely related to fresh and recency news events. One such information, may be used by search engines to promote a specific type of documents, according to the category of the query. Furthermore, we were also able to conclude that $74.2 \%$ of the temporal search sessions were initiated with queries having temporal expressions, showing that users are mostly aware of the target 
time of their information need, which is particularly true for recent dates. Our final analysis on user search satisfaction, shows that users are noticeably more satisfied in search sessions where queries have a temporal expression. This confirms the importance of temporal expressions and allows us to conclude that users search experience may benefit from being suggested likely related time periods to their initial query or shown user temporal interfaces, such as timelines.

\section{CONCLUSIONS}

In this paper we investigated the use of temporal expressions in web queries on Persian web search. To conduct this study, we used two different datasets. First, we resorted to a two-year log of a Persian search engine consisting of $27 \mathrm{M}$. Second, we rely on the above collection to select a representative sample of 10.000 queries. This enabled us to understand whether temporal expressions are or not, associated to a determined type of query category. Overall, the results obtained are in line with the work of Nunes et al. [20] and confirm that only a tiny short of Persian queries contain temporal expressions, which given the huge number of queries issued by users today, may still represent a huge quantity. Based on this, we were able to confirm that temporal expressions are mostly used within a short number of categories and that most of the temporal expressions used are related to the present time. Our analysis also revealed that $74.2 \%$ of temporal search sessions, were initiated with a temporal expression showing users awareness of his or her preferred time point(s). Finally, we were able to study that the use of temporal expressions in web searches strongly increases users' satisfaction. Compared to non-temporal search sessions, users' satisfaction is $41.3 \%$ higher when there is a query with temporal expressions in search sessions. This may be considered a noticeable number. As for future work, we plan to study how search engines should leverage explicit temporal expressions to improve search results effectiveness. We plan to do this by using the content of clicked pages and their relationship with temporal expressions. Finally, we also plan to study the use of implicit temporal expressions in Persian web searches in line with the stated-of-the-art.

\section{ACKNOWLEDGMENTS}

This research was supported by Persian native search engine program from Iran Tele-communication Research Center (ITRC). It was also partially funded by the ERDF through the COMPETE 2020 Programme within project POCI-01-0145-FEDER-006961, and by National Funds through the FCT as part of project UID/EEA/50014/2013.

\section{REFERENCES}

[1] AleAhmad, A., Zahedi, M., Rahgozar, M., \& Moshiri, B. (2016). irBlogs: A standard collection for studying Persian bloggers. Computers in Human Behavior, 57, 195-207.

[2] Arikan, I., Bedathur, S., \& Berberich, K. (2009). Time will tell: Leveraging temporal expressions in Information Retrieval. In WSDM'09.
[3] Baeza-Yates, R. (2005). Searching the Future. In S. Dominich, I. Ounis, \& J.-Y. Nie (Ed.), MFIR2005

[4] Bendersky, Michael, and W. Bruce Croft. (2009). Analysis of long queries in a large scale search log. In WSCD'19.

[5] Berberich, K., \& Bedathur, S. (2013). Temporal Diversification of Search Results. In TAIA@SIGIR'13.

[6] Berberich, K., Bedathur, S., Alonso, O., \& Weikum, G. (2010). A Language Modeling Approach for Temporal Information Needs. In ECIR'10.

[7] Campos, R., Dias, G., and Jorge, A. (2011). What is the Temporal Value of Web Snippets. In TempWeb@WWW'11, pp. 9-16.

[8] Campos, R., Dias, G., Jorge, A.M, and Jatowt, A. (2014). Survey of Temporal Information Retrieval and Related Applications. In ACM Computing Surveys. Vol 47(2): Article 15, pp 1-41

[9] Campos, R., Dias, G., Jorge, A. M., \& Nunes, C. (2012), Enriching temporal query understanding through date identification: how to tag implicit temporal queries? In TempWeb@WWW'12, 4148.

[10] Campos, R., Dias, G., Jorge, A. and Nunes, C. (2016). GTE-Rank: a Time-Aware Search Engine to Answer Time-Sensitive Queries. In Information Processing \& Management Journal. Vol 52(2), pp 273-298.

[11] Eickhoff, C., Teevan, J., White, R., \& Dumais, S. (2014). Lessons from the journey: a query $\log$ analysis of within-session learning. In WSDM'14, 223-232.

[12] Feild, H. A., Allan, J., \& Jones, R. (2010). Predicting searcher frustration. In SIGIR'10. 34-41.

[13] Fleiss, J. L. (1971). Measuring Nominal Scale Agreement Among many Raters. In Psychological Bulletin, 76(5), 378-382.

[14] Kanhabua, N., \& Nørvåg, K. (2010). Determining Time of Queries for Re-Ranking Search Results. In ECDL'10, 261-272.

[15] Kanhabua, N., \& Nørvåg, K. (2012). Learning to Rank Search Results for Time-Sensitive Queries. In CIKM'12, 2463-2466.

[16] Mansouri, B., Zahedi, M. S., Campos, R., Farhoodi, M., and Rahgozar,M. (2018). ParsTime: Rule-Based Extraction and Normalization of Persian Temporal Expressions. In ECIR'18.

[17] Mansouri, B., Zahedi, M. S.,Rahgozar,M.,and Campos, R. (2017). Detecting Seasonal Queries Using Time Series and Content Features. In ICTIR'17, 1-4.

[18] Mansouri, B., Zahedi, M. S., Rahgozar, M., Oroumchian, F., Campos, R. (2017). Learning Temporal Ambiguity in Web Search Queries. In CIKM'17, 6-10.

[19] Metzler, D., Jones, R., Peng, F., and Zhang, R. (2009). In SIGIR'09, 700-701.

[20] Nunes, S., Ribeiro, C., \& David, G. (2008). Use of temporal expressions in web search. In ECIR'08, 580-584

[21] Rieh, S. Y. (2006). Analysis of multiple query reformulations on the web: The interactive information retrieval context. In Information Processing \& Management Journal. 42(3), 751-768.

[22] Strötgen, J., Armiti, A., Van Canh, T., Zell, J., \& Gertz, M. (2014). Time for more languages: Temporal tagging of Arabic, Italian, Spanish, and Vietnamese. In TALIP '14

[23] TimeML Working Group. (2009). Guidelines for temporal expression annotation for English for tempeval.

[24] Verhagen, M., Mani, I., Sauri, R., Knippen, R., Jang, S. B., Littman,Pustejovsky, J. (2005). Automating temporal annotation with TARSQI. In ACL'05, 81-84.

[25] Zahedi, M., Aleahmad, A., Rahgozar, M., Oroumchian, F., \& Bozorgi, A. (2017). Time sensitive blog retrieval using temporal properties of queries. Journal of Information Science, 43(1), 103121

[26] Zahedi, M. S., Mansouri, B., Moradkhani, S., Farhoodi, M., \& Oroumchian, F. (2017). How questions are posed to a search engine? An empiricial analysis of question queries in a large scale Persian search engine log. In ICWR'17, 84-89. 